\title{
Evaluation of Non-invasive Fibrosis Markers in Predicting Esophageal Variceal Bleeding
}

\author{
Sami Cifci' and Nergiz Ekmen ${ }^{2}$ \\ ${ }^{1}$ Department of Gastroenterology, İstanbul Basaksehir Cam-Sakura City Hospital, Istanbul, 'Department of Gastroenterology Gazi \\ University Medical Faculty, Ankara, Turkey
}

Background/Aims: Esophageal variceal bleeding (EVB) is an important cause of mortality and morbidity in liver cirrhosis. In this study, we aimed to predict the possibility of EVB in patients with cirrhosis using a non-invasive score.

Methods: A total of 359 patients with cirrhosis were divided into two groups based on the presence or absence of EVB. ChildTurcotte-Pugh (CTP) score, a model for end-stage liver disease, aspartate aminotransferase to alanine aminotransferase ratio, aspartate aminotransferase to platelet ratio index (APRI), fibrosis-4-index (FIB-4), aspartate aminotransferase to alanine aminotransferase ratio/platelet ratio index (AARPRI), and S-index were measured for all participants. Receiver operating characteristic curves were obtained for all parameters, and the optimal cut-off value was determined in predicting EVB.

Results: In patients with EVB, the number of platelets (PLT) were low $(p<0.001)$ and APRI, AARPRI, FIB-4, and S-index were significantly higher than those in patients without EBV. APRI, AARPRI, FIB-4, PLT, and S-index were statistically significant predictors of EVB $(p<0.05)$.

Conclusions: FIB-4 and AARPRI, which are non-invasive markers of fibrosis, can be used to predict EVB. In addition, the $66.510^{9} / \mathrm{L}$ cut-off value for PLT is important for EVB. Clin Endosc 2021;54:857-863

Key Words: Bleeding; Esophageal varices; Liver cirrhosis; Non-invasive biomarkers; Platelet

\section{INTRODUCTION}

Liver cirrhosis (LC) occurs because of widespread hepatocyte necrosis, development of regenerative nodules, fibrous tissue hyperplasia, and increased intrahepatic vascular pressure due to long-term and recurrent damage of one or more factors. Portal hypertension ( $\mathrm{PH})$, which develops as a result of increased intrahepatic vascular resistance in cirrhosis, results from the combination of damage in hepatic sinusoids and dis-

Received: December 20, 2020 Revised: January 10, 2021

Accepted: January 10, 2021

Correspondence: Sami Cifci

Department of Gastroenterology, İstanbul Basaksehir Cam-Sakura City Hospital, Başakşehir Mahallesi G-434 Caddesi No: 2L Başakşehir, İstanbul 34480, Turkey Tel: +90-533-332-2077, Fax: +90-212-909-6000, E-mail: samicifci@gmail.com ORCID: https://orcid.org/0000-0003-0226-6825

cc This is an Open Access article distributed under the terms of the Creative Commons Attribution Non-Commercial License (http://creativecommons.org/ licenses/by-nc/3.0) which permits unrestricted non-commercial use, distribution, and reproduction in any medium, provided the original work is properly cited. ruption of the balance between vasodilator and vasoconstrictor agents. ${ }^{1}$

Normal portal pressure gradient values range from 1 to 5 $\mathrm{mmHg}$, and values greater than $5 \mathrm{mmHg}$ indicate the presence of $\mathrm{PH}^{2}{ }^{2} \mathrm{~A}$ wide range of spontaneous portosystemic shunts and consequently esophageal varices (EV) may also occur as complications of long-standing $\mathrm{PH}$ in patients with LC. ${ }^{3}$ The prevalence of gastroesophageal varices in LC is approximately $50 \%$ and is associated with the severity of liver disease. Esophageal variceal bleeding (EVB) occurs at a rate of $5 \%$ annually and is associated with a high mortality rate (15-25\% in 6 weeks). ${ }^{4,5}$

Evaluation of the hepatic venous pressure gradient (HVPG) is the gold standard for diagnosing and measuring the degree of $\mathrm{PH}^{6,7}$ The most common method used to detect EV is endoscopic examination; however, it may not be cost-effective to perform EV screening in clinical practice because it is an invasive procedure and less than $50 \%$ of patients with cirrhosis have $\mathrm{EV}^{4}$ 
Evaluating the severity of $\mathrm{PH}$ and $\mathrm{EV}$ is essential for the management and prognosis of the disease in patients with cirrhosis. There are some adversities with these methods. For example, HVPG measurement can only be performed in specialized centers and endoscopic examination to detect EV is not a risk-free procedure. However, since EV development results from liver fibrosis and is due to increased intrahepatic resistance, non-invasive fibrosis markers (NFM) can be useful in detecting EV. Regarding this, aspartate aminotransferase to platelet ratio index (APRI) and fibrosis-4-index (FIB-4) are recommended and validated by the World Health Organization guidelines for evaluating hepatic fibrosis. ${ }^{8}$ Owing to their easy, non-invasive, simple, and practical methods for detecting hepatic fibrosis, the model for end-stage liver disease (MELD), aspartate aminotransferase to alanine aminotransferase ratio (AAR), APRI, FIB-4, fibrosis index, and King score have been

Table 1. Comparison Clinicodemographic and Laboratory Parameters in Study Population

\begin{tabular}{|c|c|c|c|}
\hline Parameters & $\begin{array}{l}\text { No esophageal varices bleeding } \\
\qquad(n=189)\end{array}$ & $\begin{array}{l}\text { Esophageal varices bleeding } \\
\qquad(n=170)\end{array}$ & $p$-value \\
\hline Age (years) & $53.83 \pm 9.75$ & $51.92 \pm 9.61$ & $0.063^{*}$ \\
\hline Gender & & & 0.206 \\
\hline Male & $143(75.7)$ & $138(81.2)$ & \\
\hline Female & $46(24.3)$ & $32(18.8)$ & \\
\hline Etiology of cirrhosis & & & NA \\
\hline Alcoholic & $25(13.2)$ & $23(14.7)$ & \\
\hline Hepatitis B virus & $82(43.4)$ & $77(45.3)$ & \\
\hline Hepatitis $\mathrm{C}$ virus & $25(13.2)$ & $24(14.1)$ & \\
\hline Cryptogenic & $21(11.1)$ & $27(15.9)$ & \\
\hline NASH & $18(9.5)$ & $12(7.1)$ & \\
\hline Autoimmune hepatitis & $5(2.6)$ & $1(0.6)$ & \\
\hline Others & $13(6.8)$ & $4(2.4)$ & \\
\hline Varices degree & & & NA \\
\hline Grade I & $34(18)$ & $0(0.0)$ & \\
\hline Grade II & $97(51.3)$ & $27(15.9)$ & \\
\hline Grade III & $58(30.7)$ & $143(84.1)$ & \\
\hline Platelets $\left(10^{9} / \mathrm{L}\right)$ & $82(24-348)$ & $56(16-219)$ & $<0.001$ \\
\hline Serum albumin $(\mathrm{g} / \mathrm{dL})$ & $3(1.5-4.8)$ & $2.9(1.6-4.5)$ & 0.733 \\
\hline Alanine aminotransferase (U/L) & $41(9-473)$ & $34.5(7-354)$ & 0.062 \\
\hline Aspartate aminotransferase (U/L) & $59(15-317)$ & $52.5(10-463)$ & 0.076 \\
\hline CTP score & $9(5-14)$ & $9(5-15)$ & 0.393 \\
\hline MELD & $15(6-33)$ & $16(6-30)$ & 0.495 \\
\hline AAR & $1.53(0.41-11.84)$ & $1.57(0.54-4.84)$ & 0.208 \\
\hline APRI & $1.98(0.13-1.33)$ & $2.41(0.36-19.61)$ & 0.002 \\
\hline AARPRI & $2.81(0.34-26.13)$ & $4.14(0.91-16.5)$ & $<0.001$ \\
\hline FIB-4 & $6.29(0.62-43.91)$ & $8.74(2.17-42.45)$ & $<0.001$ \\
\hline S-index & $78.35(8.80-3172)$ & $94.10(4.38-2126)$ & 0.025 \\
\hline
\end{tabular}

Data are expressed as mean $\pm \mathrm{SD}$, number (\%), or median (range).

$\mathrm{AAR}$, aspartate aminotransferase to alanine aminotransferase ratio; AARPRI, aspartate aminotransferase to alanine aminotransferase ratio/platelet ratio index; APRI, aspartate aminotransferase-to-platelet-ratio index; CTP: Child-Turcotte-Pugh score; NASH, nonalcoholic steatohepatitis; FIB-4, fibrosis-4 index; MELD, model for end-stage liver disease; NA, not applicable.

${ }^{*} p$-value from Student's $t$-test. All other $p$-values are obtained from Mann-Whitney's $U$-test. 
evaluated for the early detection of EV. ${ }^{9-14}$

In this study, we aimed to predict the possibility of EVB using non-invasive fibrosis indicators to reduce the complications associated with the number of endoscopic scans, increase the cost-effectiveness, and determine the best time for invasive procedures.

\section{PATIENTS AND METHODS}

\section{Study population}

This study was conducted between 2015 and 2020 by scanning the files of patients followed-up for LC, online hospital data, and endoscopy records in the gastroenterology clinic of the institution. A total of 413 patients over the age of 18 years who had cirrhosis and detected EV in upper gastrointestinal endoscopy were included in the preliminary examination. Data on age, sex, cirrhosis etiology, MELD and Child-Turcotte-Pugh (CTP) classification scores, platelet (PLT) and biochemical results, endoscopically defined EV degrees, EVB history of the previous year were recorded. A total of 54 patients with portosystemic shunt, previous gastrointestinal surgery, liver metastasis, hepatocellular carcinoma, portal, hepatic and splenic vein thrombosis, myeloproliferative disease, pre-splenectomy, and those with a history of transjugular intrahepatic portosystemic shunt were excluded from the study.

\section{Endoscopic examination}

Endoscopies were performed by a gastroenterologist using Fujinon EG-580RD (Fujifilm Europe, Düsseldorf, Germany) brand gastroscopy devices. Varices grading was defined as grade I, II, and III. ${ }^{15}$

\section{Calculation of non-invasive fibrosis markers}

The following formulas were used to calculate the investigated non-invasive markers:

1) $\mathrm{AAR}=$ aspartate aminotransferase (AST)/alanine aminotransferase (ALT) ratio

2) FIB- $4=($ year of age $\times$ AST $) /($ PLT $\times$ the square root of ALT)

3) $\mathrm{APRI}=(\mathrm{AST} /$ upper limit of normal $) \times 100 / \mathrm{PLT}\left(10^{9} / \mathrm{L}\right)$

5) S-index $=1000 \times$ gamma glutamyl transferase $/($ PLT $\times$ albumin $^{2}$ )

6) aspartate aminotransferase to alanine aminotransferase ratio/platelet ratio index $(\mathrm{AARPRI})=\mathrm{AAR} /(\mathrm{PLT}$ count $\left.\left(10^{9} / \mathrm{L}\right) / 150\right)$

\section{Statistical analysis}

All data were analyzed using SPSS Statistics version 21 (IBM
Co., Armonk, NY, USA). The consistency of continuous variables to normal distribution was evaluated using visual (histogram and probability plots) and analytical methods (Kolmogorov-Smirnov/Shapiro-Wilk tests).

Categorical variables are reported as numbers, percentages, normally distributed data as arithmetic means and standard deviations, and skewed-distributed data as median (minimum-maximum) values. An independent sample t-test was used for the comparative analysis between two independent groups of normally distributed data, and the Mann-Whitney $\mathrm{U}$ test was used in the non-compliant data. In the comparative analysis for categorical variables between independent groups, the Pearson's or Fisher's test was chosen from the chi-square $\left(\chi^{2}\right)$ test. Receiver operating characteristic curve (ROC) analysis was performed to calculate the esophageal bleeding predictive value of NFM (APRI, AARPRI, FIB-4, AAR, and S-index), CTP score, and MELD. The area under the ROC curve (AUC) results were considered as follows: $0.9-1$, excellent; $0.8-0.9$, good; 0.7-0.8, fair; 0.6-0.7, poor; and 0.5-0.6, failed. ${ }^{16}$ The results following ROC analysis, AUC and cut-off values, sensitivity, and specificity of these cut-off values, likelihood ratio (LR), odds ratio (OR), positive predictive value (PPV), and negative predictive value (NPV) were also presented. A $p$-value $<0.05$ was considered statistically significant.

\section{Ethical approval}

The study protocol was carried out with the approval of the Ethics Committee of the Ordu Training and Research Hospital, Ordu (Turkey) (No: 16/2018).

\section{RESULTS}

\section{Demographic characteristics of the study population}

In the analysis based on the presence of bleeding, no significant difference was found between the groups in terms of age and sex $(p>0.05)$. The most common etiologic factor of cirrhosis in both groups was hepatitis B virus (Table 1).

In the group with EVB, grade I esophageal bleeding was not detected, while grade III esophageal varices were found in $84.1 \%(n=143)$ of cases.

\section{Prediction of EVB with non-invasive markers}

There was no significant difference between the groups in terms of albumin, AST, ALT, CTP score, MELD, and AAR $(p>0.05)$. The number of PLT was significantly lower in the group with bleeding $(p<0.001)$. APRI, AARPRI, FIB-4, and $S$-index were significantly higher in the group with bleeding $(p=0.002,<0.001,<0.001$, and 0.025 , respectively). 
Table 2. Performance of Non-Invasive Markers for Prediction of Esophageal Variceal Bleeding

\begin{tabular}{|c|c|c|c|c|c|c|c|c|c|c|c|}
\hline Variable & $\begin{array}{c}\text { AUC } \\
(95 \% \mathrm{CI})\end{array}$ & $p$-value & $\begin{array}{l}\text { Cut-off } \\
\text { value }\end{array}$ & $\begin{array}{c}\text { Sensitivity } \\
(\%)\end{array}$ & $\begin{array}{c}\text { Specificity } \\
(\%)\end{array}$ & $\begin{array}{r}\text { PPV } \\
(\%)\end{array}$ & $\begin{array}{l}\text { NPV } \\
(\%)\end{array}$ & $\begin{array}{c}\text { Diagnostic } \\
\text { accuracy (\%) }\end{array}$ & LR + & LR - & $\begin{array}{c}\text { OR } \\
(\mathrm{EV} \text { vs. } \mathrm{NEV})\end{array}$ \\
\hline${ }^{*} \mathrm{CTP}$ & $\begin{array}{c}0.52 \\
(0.46-0.58)\end{array}$ & 0.398 & $\geq 8.5$ & 60.60 & 44.30 & 49.28 & 55.33 & 51.81 & 1.08 & 0.9 & $\begin{array}{c}1.20 \\
(0.79-1.83)\end{array}$ \\
\hline${ }^{\star}$ MELD & $\begin{array}{c}0.52 \\
(0.46-0.58)\end{array}$ & 0.496 & $\geq 14.5$ & 57.10 & 49.10 & 50.00 & 55.76 & 52.65 & 1.11 & 0.88 & $\begin{array}{c}1.26 \\
(0.83-1.91)\end{array}$ \\
\hline${ }^{*} \mathrm{AAR}$ & $\begin{array}{c}0.53 \\
(0.47-0.59)\end{array}$ & 0.208 & $\geq 1.51$ & 57.10 & 49.20 & 50.06 & 56.02 & 52.92 & 1.12 & 0.87 & $\begin{array}{c}1.29 \\
(0.85-1.95)\end{array}$ \\
\hline APRI & $\begin{array}{c}0.59 \\
(0.53-0.65)\end{array}$ & 0.002 & $\geq 2.01$ & 60.00 & 52.10 & 52.85 & 59.04 & 55.71 & 1.25 & 0.77 & $\begin{array}{c}1.61 \\
(1.06-2.45)\end{array}$ \\
\hline AARPRI & $\begin{array}{c}0.68 \\
(0.63-0.74)\end{array}$ & $<0.001$ & $\geq 3.02$ & 70.00 & 58.10 & 59.50 & 67.92 & 63.23 & 1.63 & 0.53 & $\begin{array}{c}3.11 \\
(2.01-4.81)\end{array}$ \\
\hline FIB-4 & $\begin{array}{c}0.63 \\
(0.57-0.69)\end{array}$ & $<0.001$ & $\geq 6.70$ & 65.20 & 57.40 & 57.22 & 64.24 & 60.45 & 1.49 & 0.62 & $\begin{array}{c}2.40 \\
(1.56-3.68)\end{array}$ \\
\hline S-index & $\begin{array}{c}0.56 \\
(0.50-0.62)\end{array}$ & 0.025 & $\geq 75.44$ & 58.20 & 48.30 & 50.25 & 56.17 & 52.92 & 1.12 & 0.87 & $\begin{array}{c}1.29 \\
(0.85-1.96)\end{array}$ \\
\hline PLT & $\begin{array}{c}0.70 \\
(0.65-0.75)\end{array}$ & $<0.001$ & $\leq 66.50$ & 62.40 & 70.30 & 64.63 & 67.18 & 66.02 & 2.03 & 0.54 & $\begin{array}{c}3.74 \\
(2.41-5.97)\end{array}$ \\
\hline
\end{tabular}

AAR, aspartate aminotransferase to alanine aminotransferase ratio; APRI, aspartate aminotransferase to platelet ratio index; AARPRI, aspartate aminotransferase to alanine aminotransferase ratio/platelet ratio index; AUC, area under the curve; CI: confidence interval; CTP: Child-Turcotte-Pugh score; EV, esophageal varices; FIB-4: fibrosis-4-index; LR, likelihood ratio; MELD: a model for end-stage liver disease; NEV, no esophageal varices; NPV, negative predictive value; OR, odds ratios, PLT, platelet; PPV, positive predictive value. ${ }^{\star}$ Statistically not significant AUC obtained from the receiver operating characteristic. 95\% CI and $p$-values from Chi-square analysis.

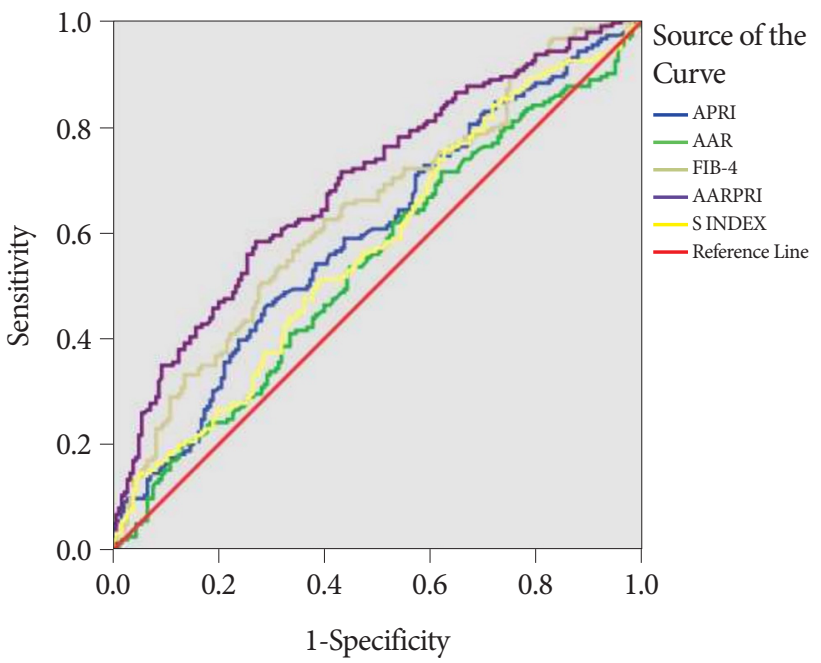

Fig. 1. Receiver operating characteristic curves for the APRI, AAR, FIB4, AARPRI, and S-index. Larger results of APRI, AAR, FIB- 4, AARPRI, and $\mathrm{S}$-index indicate more diagnostic positive tests for esophageal variceal bleeding. AAR, aspartate aminotransferase to alanine aminotransferase ratio; APRI, aspartate aminotransferase-to-platelet-ratio index; AARPRI, aspartate aminotransferase to alanine aminotransferase ratio/platelet ratio index; FIB-4, fibrosis-4 index.

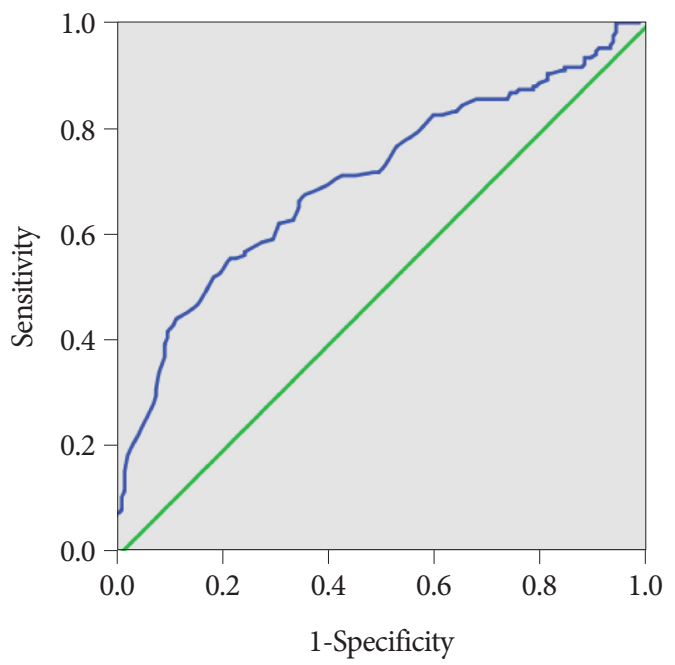

Fig. 2. Receiver operating characteristic curves for the platelet. A small number of platelets indicate a more diagnostic positive test for esophageal varices bleeding. 
The predictive power of the CTP score, MELD, AAR, APRI, AARPRI, FIB-4, PLT, and S-index were evaluated by ROC analysis. AUC values and significance obtained from ROC analysis are presented in Table 2. MELD, CTP score, and AAR were not significant predictors of variceal bleeding $(p>0.05)$. The AUC values obtained for FIB-4 (AUC $=0.63$ ), APRI (AUC $=0.59)$, and S-index $(\mathrm{AUC}=0.56)$ were significant but weak (Fig. 1). The highest significant AUC value was obtained for PLT (AUC=0.7) (Fig. 2) and AARPRI (AUC=0.68). Sensitivity, specificity, + LR, - LR, PPV, NPV, and OR of the cut-off values obtained for the parameters found significant by ROC analysis were calculated. It was observed that the values of PLT $\leq 66.510^{9} / \mathrm{L}$ were better than other parameters (sensitivity, 62\%; specificity 70\%; + LR, 2.03; PPV, 64.3\%; NPV, 67.18; OR, 3.74).

\section{DISCUSSION}

EVB is a fatal complication of cirrhotic PH. However, there is no apparent clinical finding for EV in most patients with cirrhosis, even during the decompensation period.

Today, clinicians are concerned with identifying some non-invasive biochemical markers with high sensitivity and specificity that are cheaper and easier to obtain to reduce the number of upper gastrointestinal endoscopies for screening and treating EV in liver patients. These non-invasive biomarkers are applied using routine laboratory tests that do not require extra cost and special devices or additional biochemical tests. ${ }^{17}$ NFM can also predict the development of EV, as $\mathrm{PH}$ is a result of increased liver vascular resistance secondary to liver fibrosis. ${ }^{18} \mathrm{Few}$ studies have investigated non-invasive approaches to estimate the risk and development of EV and $\mathrm{EVB}$, and this issue remains controversial. ${ }^{19-21}$

While Iwata et al., reported that AAR was related to the severity of esophageal varices, ${ }^{22}$ there are some publications in the literature indicating that the AAR index has limited predictive value for severe $\mathrm{EV}^{23}$ In our study, the AAR index did not show a significant difference between the groups.

The APRI and FIB-4 index are two classic non-invasive scores with good diagnostic efficacy for cirrhosis. ${ }^{24,25}$ Zhang et al., determined that the AUC value of the APRI (0.729) was higher than that of the other three indices (AAR, FIB-4, and $S$-index) in determining the presence and severity of $\mathrm{EV}$, and the APRI score greater than 1.4 can be used as a reference indicator for the early intervention of severe $\mathrm{EV}^{23} \mathrm{In}$ addition, the APRI score was shown to be an independent predictor of recurrent EVB in a case-control study. ${ }^{26}$ In a study that included only alcoholic cirrhotic patients, the average APRI values were significantly higher in the EVB group. ${ }^{27}$ In our study, the
APRI index was significantly higher in the EVB group than in the non-EVB group $(p=0.002)$. However, the APRI score was found to be poorly associated with the prediction of EVB (OR, 1.61).

In the study by Zhang et al., the FIB-4 index was an independent predictor of $\mathrm{EV}$, and the AUROC value was determined to be $0.64 .^{23}$ In a large meta-analysis, the AUC value of FIB-4 was reported as 0.77 in predicting the presence of $\mathrm{EV}^{28}$ A study conducted by Kraja et al. ${ }^{29}$ defined the FIB-4 score as a strong and significant predictor of $\mathrm{EV}$ presence. In the same study, the FIB-4 score was defined as an important predictor for EV at a 3.23 cut-off value (AUC, 0.66 ) and a weak predictor for EVB at a 5.02 cut-off value (AUC, 0.51 ). They interpreted FIB-4 as the most effective NFM of the liver that can be used as the first screening tool for cirrhotic patients. ${ }^{29}$ In a study of patients with alcoholic cirrhosis, the mean FIB-4 score was found to be significantly higher in EVB group than in the non-EVB group (8.0 and 3.9, respectively), thereby predicting EVB with a diagnostic accuracy of $63.86 \%{ }^{27}$ Similarly, in the current study, we also found that the FIB-4 score was significantly higher in the EBV group than in the non-EVB group $(p<0.001)$. In addition, FIB-4 had a diagnostic accuracy rate of $60.45 \%$ at a cut-off value of 6.70 . The predictive power of FIB-4 of EVB was found to be higher than the APRI score (OR, 2.40 and 1.61, respectively).

According to the Baveno VI consensus criteria, it was stated that endoscopic examination was not required in patients with transient elastography value less than $20 \mathrm{kPa}$ and platelet value more than $150 \times 10^{9} / \mathrm{L}$ and annual PLT level monitoring was recommended. ${ }^{30}$ Sarangapani et al., stated that a platelet count lower than $150 \times 10^{9} / \mathrm{L}$ was an independent predictor of the presence of $\mathrm{EV}^{31}$ Madonia et al., associated thrombocytopenia with recurrent variceal bleeding and stated that it was below the $80 \times 10^{9} / \mathrm{L}$ level as the lower limit. ${ }^{32}$ In the current study, the number of platelets, determined as the strongest predictor of EVB (AUC, 0,70; OR, 3,74), had a $62 \%$ sensitivity and 70\% specificity at a cut-off value of $66.5 \times 10^{9} / \mathrm{L}$.

In a study where the S-Index and FIB-4 score were evaluated by ROC analysis for the predictive power of EVB, the S-index was found to be stronger than the FIB- 4 score (AUC, 0.695 and 0.673 , respectively). However, it has been stated that $\mathrm{EV}$ is not an independent predictor (all $p>0.01$ ). ${ }^{23}$ In our study, the $S$-index was found to be significantly higher in patients with EVB than in patients without EVB $(p=0.025)$. In addition, the power of the S-index to predict EVB was very weak (AUC, 0.56; OR, 1.29).

In a limited number of studies in which the AARPRI score was examined, a significant relationship was found between the liver fibrosis rate and the AARPRI score. ${ }^{33,34}$ However, to the best of our knowledge, no study has evaluated the relation- 
ship between EV and AARPRI scores. In our study, AARPRI was found to have moderate power to predict the likelihood of EVB (AUC, 0.68; OR, 3,11).

In addition, to predict the likelihood of EVB, AARPRI was found to have a $63.33 \%$ diagnostic accuracy rate with a 1.63 likelihood at a 3.02 cut-off value (70\% sensitivity and 58\% specificity).

There were some limitations in this study. First, due to the retrospective nature of the study, we could not investigate the predictive values of these markers for EVB in patients without a previous history of EVB. Second, since the treatment records of patients were not fully available, it was not possible to distinguish patients who received prophylactic treatment for EVB.

In conclusion, the current study showed that FIB-4, AARPRI, and PLT as non-invasive liver fibrosis markers will contribute to our clinical knowledge in predicting EVB. However, larger studies are needed to implement a routine use of these markers in clinical practice. We hope that this study will guide future studies on the use of non-invasive methods as a screening method to predict EVB.

Conflicts of Interest

The authors have no potential conflicts of interest.

Funding

None.

Author Contributions

Conceptualization: Sami Cifci, Nergiz Ekmen

Data curation: SC, NE

Formal analysis: SC, NE

Investigation: SC, NE

Methodology: SC, NE

Project administration: SC, NE

Ono Supervision: SC, NE

Validation: SC, NE

Writing-original draft: SC, NE

Writing-review\&editing: SC, NE

ORCID

Sami Cifci:

Nergiz Ekmen:

https://orcid.org/0000-0003-0226-6825 https://orcid.org/0000-0002-7921-3169

\section{REFERENCES}

1. Rahimi RS, Rockey DC. Complications and outcomes in chronic liver disease. Curr Opin Gastroenterol 2011;27:204-209.

2. Ripoll C, Groszmann R, Garcia-Tsao G, et al. Hepatic venous pressure gradient predicts clinical decompensation in patients with compensated cirrhosis. Gastroenterology 2007;133:481-488.

3. de Franchis R, Baveno VI Faculty. Expanding consensus in portal hypertension: report of the Baveno VI Consensus Workshop: stratifying risk and individualizing care for portal hypertension. J Hepatol 2015;63:743752.
4. Garcia-Tsao G, Sanyal AJ, Grace ND, Carey W, Practice Guidelines Committee of the American Association for the Study of Liver Diseases, Practice Parameters Committee of the American College of Gastroenterology. Prevention and management of gastroesophageal varices and variceal hemorrhage in cirrhosis. Hepatology 2007;46:922-938.

5. Garcia-Tsao G, Abraldes JG, Berzigotti A, Bosch J. Portal hypertensive bleeding in cirrhosis: risk stratification, diagnosis, and management: 2016 practice guidance by the American Association for the study of liver diseases. Hepatology 2017;65:310-335.

6. Gracia-Sancho J, Marrone G, Fernández-Iglesias A. Hepatic microcirculation and mechanisms of portal hypertension. Nat Rev Gastroenterol Hepatol 2019;16:221-234.

7. Karagiannakis DS, Voulgaris T, Siakavellas SI, Papatheodoridis GV, Vlachogiannakos J. Evaluation of portal hypertension in the cirrhotic patient: hepatic vein pressure gradient and beyond. Scand J Gastroenterol 2018;53:1153-1164.

8. Stasi C, Milani S. Non-invasive assessment of liver fibrosis: between prediction/prevention of outcomes and cost-effectiveness. World J Gastroenterol 2016;22:1711-1720.

9. Madhotra R, Mulcahy HE, Willner I, Reuben A. Prediction of esophageal varices in patients with cirrhosis. J Clin Gastroenterol 2002;34:8185.

10. Giannini EG, Zaman A, Kreil A, et al. Platelet count/spleen diameter ratio for the noninvasive diagnosis of esophageal varices: results of a multicenter, prospective, validation study. Am J Gastroenterol 2006;101:25112519.

11. Sebastiani G, Tempesta D, Fattovich G, et al. Prediction of oesophageal varices in hepatic cirrhosis by simple serum non-invasive markers: results of a multicenter, large-scale study. J Hepatol 2010;53:630-638.

12. Tafarel JR, Tolentino LHL, Correa LM, et al. Prediction of esophageal varices in hepatic cirrhosis by noninvasive markers. Eur J Gastroenterol Hepatol 2011;23:754-758.

13. Deng H, Qi X, Peng Y, et al. Diagnostic accuracy of APRI, AAR, FIB-4, FI, and king scores for diagnosis of esophageal varices in liver cirrhosis: a retrospective study. Med Sci Monit 2015;21:3961-3977.

14. Hong W-D, Zhu Q-H, Huang Z-M, et al. Predictors of esophageal varices in patients with HBV-related cirrhosis: a retrospective study. BMC Gastroenterol 2009;9:11.

15. Tripathi D, Stanley AJ, Hayes PC, et al. U.K. guidelines on the management of variceal haemorrhage in cirrhotic patients. Gut 2015;64:16801704.

16. Obuchowski NA. Receiver operating characteristic curves and their use in radiology. Radiology 2003;229:3-8.

17. Procopet B, Berzigotti A. New tools for the noninvasive assessment of cirrhosis. Curr Hepatology Rep 2014;13:14-23.

18. Stefanescu H, Procopet B. Noninvasive assessment of portal hypertension in cirrhosis: liver stiffness and beyond. World J Gastroenterol 2014;20:16811-16819.

19. Wang X, Wang B-M, Li G, Li Z-J, Chen C, Piao M-Y. A clinical prediction model and its application for bleeding in chronic liver failure patients with esophageal varices. Eur Rev Med Pharmacol Sci 2013;17:3046-3055

20. Hanafy AS, Badawi R, Basha MAA, et al. A novel scoring system for prediction of esophageal varices in critically ill patients. Clin Exp Gastroenterol 2017;10:315-325.

21. Wang X-K, Wang P, Zhang Y, Qi S-L, Liu W, Wang G-C. A study on two kinds of scoring models in predicting the degree of esophageal varices and bleeding. Eur Rev Med Pharmacol Sci 2020;24:3876-3881.

22. Iwata Y, Enomoto H, Sakai Y, et al. Elevation of the AST to ALT ratio in association with the severity of esophageal varices in patients with HCV-related compensated liver cirrhosis. Hepatogastroenterology 2013;60:149-152

23. Zhang F, Liu T, Gao P, Fei S. Predictive value of a noninvasive serological hepatic fibrosis scoring system in cirrhosis combined with oesophageal 
varices. Can J Gastroenterol Hepatol 2018;2018:7671508.

24. Shin WG, Park SH, Jang MK, et al. Aspartate aminotransferase to platelet ratio index (APRI) can predict liver fibrosis in chronic hepatitis B. Dig Liver Dis 2008;40:267-274.

25. Vallet-Pichard A, Mallet V, Pol S. FIB-4: a simple, inexpensive and accurate marker of fibrosis in HCV-infected patients. Hepatology 2006;44:769;author reply769-770

26. Vanbiervliet G, Giudicelli-Bornard S, Piche T, et al. Predictive factors of bleeding related to post-banding ulcer following endoscopic variceal ligation in cirrhotic patients: a case-control study. Aliment Pharmacol Ther 2010;32:225-232.

27. Kothari HG, Gupta SJ, Gaikwad NR, Sankalecha TH, Samarth AR. Role of non-invasive markers in prediction of esophageal varices and variceal bleeding in patients of alcoholic liver cirrhosis from central India. Turk J Gastroenterol 2019;30:1036-1043.

28. Deng H, Qi X, Guo X. Diagnostic accuracy of APRI, AAR, FIB-4, FI, king, lok, forns, and fibroindex scores in predicting the presence of esophageal varices in liver cirrhosis: a systematic review and meta-analysis. Medicine (Baltimore) 2015;94:e1795

29. Kraja B, Mone I, Akshija I, Koçollari A, Prifti S, Burazeri G. Predictors of esophageal varices and first variceal bleeding in liver cirrhosis patients. World J Gastroenterol 2017;23:4806-4814.

30. Cardenas A, Mendez-Bocanegra A. Report of the Baveno VI consensus workshop. Ann Hepatol 2016;15:289-290.

31. Sarangapani A, Shanmugam C, Kalyanasundaram M, Rangachari B, Thangavelu P, Subbarayan JK. Noninvasive prediction of large esophageal varices in chronic liver disease patients. Saudi J Gastroenterol 2010;16:38-42.

32. Madonia S, D’Amico G, Traina M, et al. Prognostic indicators of successful endoscopic sclerotherapy for prevention of rebleeding from oesophageal varices in cirrhosis: a long-term cohort study. Dig Liver Dis 2000;32:782-791.

33. Wang H-W, Peng C-Y, Lai H-C, et al. New noninvasive index for predicting liver fibrosis in Asian patients with chronic viral hepatitis. Sci Rep 2017;7:3259.

34. PK MM, Sha MdF, Pervin R, et al. Investigating the efficiency of APRI, FIB-4, AAR and AARPRI as noninvasive markers for predicting hepatic fibrosis in chronic hepatitis B patients in Bangladesh. The Open Microbiology Journal 2019;13:34-40. 